\title{
Study of Single-Charge Polarization on two Charge Qubits Integrated onto a Double Single-Electron Transistor Readout
}

\author{
Yoshiyuki Kawata $^{1,4}$, Satoshi Nishimoto ${ }^{1}$, Yoshishige Tsuchiya ${ }^{1,4}$, Shunri Oda ${ }^{1,4}$, and Hiroshi Mizuta ${ }^{2,3,4}$ \\ ${ }^{1}$ Quantum Nanoelectronics Research Center, Tokyo Institute of Technology, \\ 2-12-1, O-okayama, Meguro-ku, Tokyo 152-8552, Japan \\ Phone: +81-5734-3854 FAX: +81-5734-2542 e-mail: kawata@neo.pe.titech.ac.jp \\ ${ }^{2}$ School of Electronics and Computer Science, University of Southampton, Southampton, UK \\ ${ }^{3}$ Department of Physical Electronics, Tokyo Institute of Technology, Tokyo, Japan \\ ${ }^{4}$ SORST-JST (Japan Science and Technology Agency)
}

\section{Introduction}

Solid-state approach offers the promising way to integrate a large number of quantum bits (qubits) to realize quantum computers. Double Quantum Dots (DQDs) have been extensively studied as a candidate for the charge qubit. Early DQD research has been done for GaAs/AlGaAs hetero-structures [1], but silicon based DQDs have recently been fabricated using the top-down fabrication technique, and a long decoherence time has been demonstrated [2]. Silicon based DQDs are also preferred because of their compatibility with the existing silicon fabrication process. Decoherence time of the qubit should be extended further for accomplishing fault tolerant quantum computation. It has also been studied to adopt nanocystalline Si DQDs deposited by VHF plasma deposition technique for realizing extremely downscaled charge qubits [3].

Another key issue is to integrate multiple charge qubits with a suitable readout device. We recently proposed multiple single-electron transistors (MSETs) [4], where SETs are connected in series, for sensing single-charge polarization over the multiple qubits. In this paper we fabricate and evaluate a double single-electron transistor (DSET) (Fig. 1) to clarify the readout scheme. In the DSET configuration, qubits operation is controlled via electrodes (G3-G7), and the gate electrodes $\mathrm{G} 1$ and $\mathrm{G} 2$ are used to manipulate the electrochemical potentials on the SET islands. We demonstrate how different single-charge configurations on a pair of DQDs are sensed by using the DSETs by using the measured electrical characteristics for the DSET and the equivalent circuit simulation.

\section{Fabrication of DSETs}

DSET was fabricated on the phosphorus doped $\left(\sim 10^{19}\right.$ $\mathrm{cm}^{-3}$ ) silicon-on-insulator (SOI) substrate with BOX thickness of $200 \mathrm{~nm}$. SEM image of fabricated DSET without qubits and with qubits are shown in Fig. 1(a) and Fig. 1(b), respectively. Lithographically defined diameter of the each island was $90 \mathrm{~nm}$ and pattern was transferred to SOI by using the electron cyclotron resonance-reactive ion etching (ECR-RIE) technique. At this fabrication process, thickness of the SOI layer is $40 \mathrm{~nm}$. After etching, thermal oxidation was done at $1000{ }^{\circ} \mathrm{C}$ to passivate the surface states and to reduce the effective thickness of the SOI down to $30 \mathrm{~nm}$. This leads to the resulting overall dot diameter of approximately $45 \mathrm{~nm}$. In this device structure, adjacent constrictions act as tunnel barriers connecting the charging islands to the source and drain electrodes.

\section{Electrical characteristics for DSETs}

Electrical measurements were carried out at $4.2 \mathrm{~K}$ for the fabricated DSET without qubits. Figure 2 and Figure 3 show the contour plots of differential conductance $\left(\partial I_{\mathrm{D}} / \partial V_{\mathrm{D}}\right)$ as a function of $V_{\mathrm{G} 1}, V_{\mathrm{D}}$ and $V_{\mathrm{G} 2}, V_{\mathrm{D}}$, respectively. Clear Coulomb blockade with different Coulomb diamond sizes can be observed in both the plots. This confirms the presence of the two islands in the fabricated structure. Contour plot of $I_{\mathrm{D}}$ as a function of $V_{\mathrm{G} 1}$ and $V_{\mathrm{G} 2}$ is shown in the Fig. 4 for the drain voltage of $500 \mu \mathrm{V}$. From this contour plot, it can be understood that number of electrons in each of the SET can be controlled individually by the gate potentials. The equivalent circuit of DSET is shown in Fig. 5 (a). Equivalent circuit capacitance values $C_{\mathrm{G} 111}=0.52 \quad \mathrm{aF}$ (G1-Island 1), and $C_{\mathrm{G} 12}=0.38 \mathrm{aF}$ (G1-Island 2), $C_{\mathrm{G} 211}=0.75$ $\mathrm{aF}$ (G2-Island 1), $C_{\mathrm{G} 212}=1.05 \mathrm{aF}$ (G2-Island 2) were extracted from the contour plot of the gates $\mathrm{G} 1$ and $\mathrm{G} 2$.

\section{Readout of single-charge polarization on two qubits}

To characterize the effect of charge polarizations in a pair of DQDs on the DSET, equivalent circuit simulation was done at $4.2 \mathrm{~K}$. The equivalent circuit of the DSET with DQDs is shown in Fig. 5 (b). Figures 6(a) and 6(b) show the simulation results for charge configurations of DQDs shown in the inset figures. Triple points in Fig.6 (b) are shifted toward right bottom direction from those points in Fig. 6 (a). It indicates that change in polarizations in the pair of DQDs shift contour plot characteristics remarkably. Effects of the various polarization condition on DSET can be easily understood from the drain current characteristics as a function of $V_{\mathrm{G} 2}$ at $V_{\mathrm{G} 1}=1.0 \mathrm{~V}$ shown in Fig. 6 (c).

\section{Conclusions}

We fabricated DSET to detect single-charge polarization on a pair of qubits independently. Operation of the individual SETs was successfully demonstrated, and the electrical characteristics measured for the DSET were validated by comparing with the equivalent circuit simulations. We also demonstrated that charge polarizations on the pair of qubits could be distinguished by using the adjacent DSET.

\section{References}

[1] T. Fujisawa et al., Physica E 21, 1046 (2004).

[2] J. Gorman et. al., Phys. Rev. Lett., 95, 090502 (2005).

[3] Y. Kawata et al., SSDM 2006, pp.812 (2006).

[4] Y. Kawata et al., to be published at SNW 2007, June (2007). 
(a)

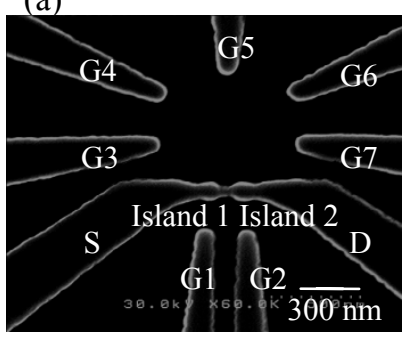

(b)

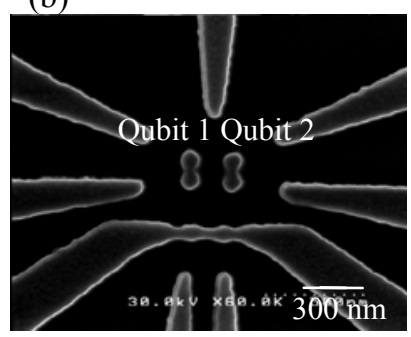

Fig. 1 SEM image of DSETs and qubits gates: (a) without qubits; (b) with qubits.

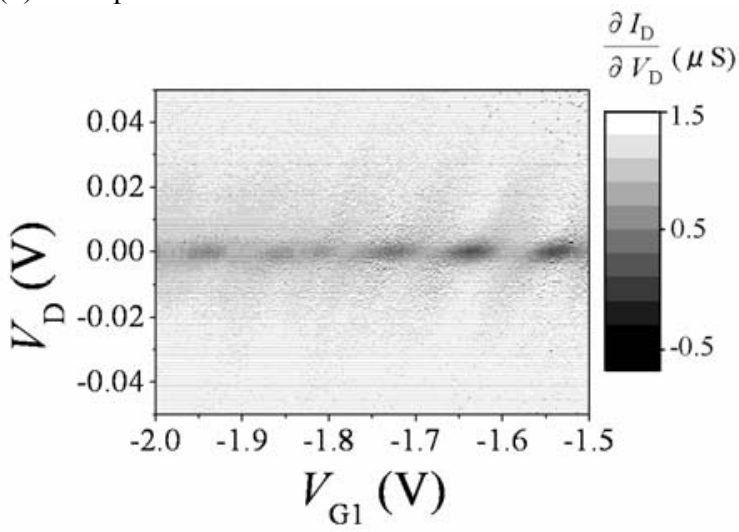

Fig. 2 Contour plot of the measured differential conductance $\left(\partial I_{\mathrm{D}} / \partial V_{\mathrm{D}}\right)$ as a function of $V_{\mathrm{G} 1}$ and $V_{D}$ at $4.2 \mathrm{~K}$.

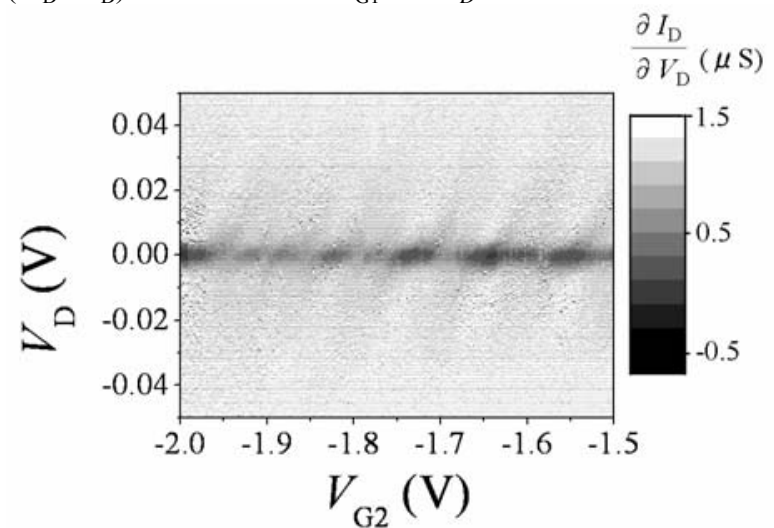

Fig. 3 Contour plot of the measured differential conductance $\left(\partial I_{\mathrm{D}} / \partial V_{\mathrm{D}}\right)$ as a function of $V_{\mathrm{G} 2}$ and $V_{D}$ at $4.2 \mathrm{~K}$.

$$
I_{\mathrm{D}}(\mathrm{nA})
$$

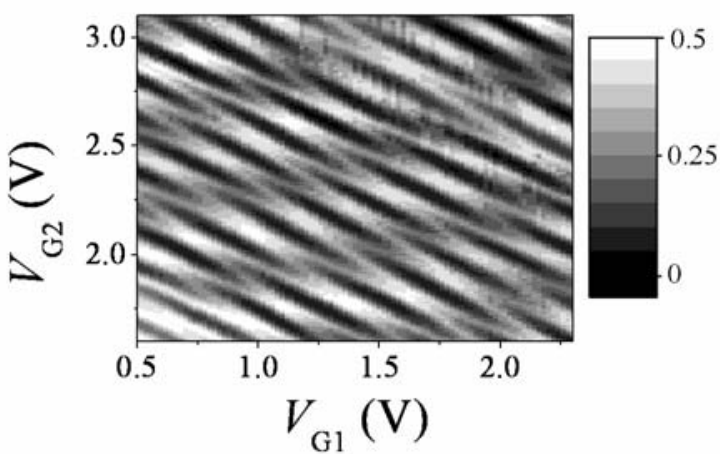

Fig. 4 Contour plot of the measured $I_{\mathrm{D}}$ as a function of $V_{\mathrm{G} 1}$ and $V_{\mathrm{G} 2}$ with $V_{\mathrm{D}}=500 \mu \mathrm{V}$ at $4.2 \mathrm{~K}$. (a)

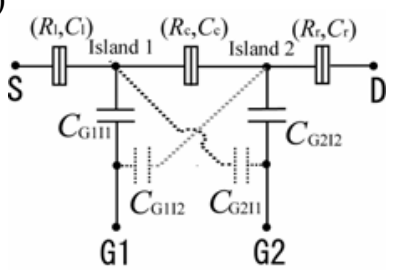

(b)

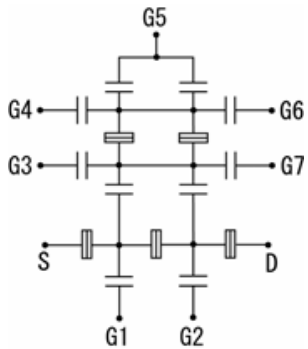

Fig. 5 Equivalent Circuits of (a) DSET; (b) DSET with qubits. Cross capacitances are abbreviated for clarity.

(a)
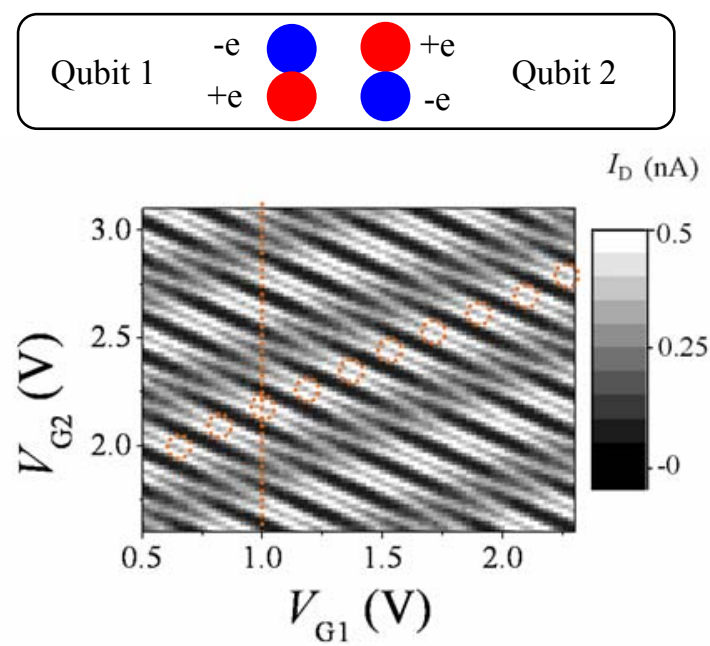

(b)
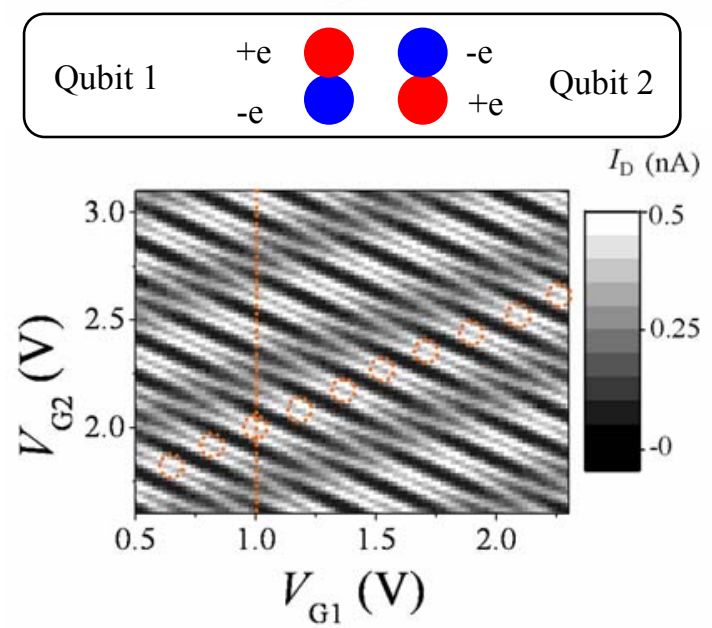

(C)

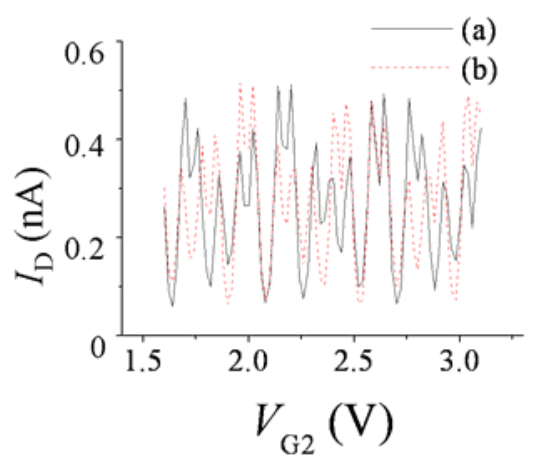

Fig. 6 (a), (b): Contour plots of the simulated $I_{\mathrm{D}}$ as a function of $V_{\mathrm{G} 1}$ and $V_{\mathrm{G} 2}$ with $V_{\mathrm{D}}=500 \mu \mathrm{V}$ at $4.2 \mathrm{~K}$ for the charge polarizations shown in inset figures. (c) $I_{\mathrm{D}}-V_{\mathrm{G} 2}$ at $V_{\mathrm{G} 1}=1.0 \mathrm{~V}$ shown as dotted lines in (a) and (b). 